\title{
EHV AC Parallel Transmission Line Calculations with Application to the Near Resonance Problem
}

\author{
A. Norton Chaston \\ nchaston@comcast.net
}

Follow this and additional works at: https://scholarsarchive.byu.edu/facpub

Part of the Electrical and Computer Engineering Commons

\section{Original Publication Citation}

Chaston, A. N. "EHV AC Parallel Transmission Line Calculations with Application to the Near Resonance Problem." power apparatus and systems, ieee transactions on PAS-88.5Part-I (1969): 627-35

\section{BYU ScholarsArchive Citation}

Chaston, A. Norton, "EHV AC Parallel Transmission Line Calculations with Application to the Near Resonance Problem" (1969). Faculty Publications. 800.

https://scholarsarchive.byu.edu/facpub/800 


\title{
EHV AC Parallel Transmission Line Calculations with Application to the Near Resonance Problem
}

\author{
A. NORTON CHASTON, SENIOR MEMBER, IEEE
}

\begin{abstract}
The use of shunt reactors with EHV transmission lines has introduced new problems, one of which is induction of high voltages on a de-energized circuit of two parallel lines. This paper presents the matrix equations used in developing a digital computer program which takes into account the effects of electromagnetic induction, electrostatic coupling, distributed lines, and transposition. The computer program may also be used to calculate the effects of shunt reactors, series capacitors, and loads. The equations can be adapted to a wide range of applications, although the examples for this paper have been limited to the near resonance problem of parallel transmission lines.
\end{abstract}

\section{INIRODCCTION}

$\mathbf{E}$ XTREME vo'tages and large currents can be developed in the de-energized circuit of EHV parallel transmission lines as a result of the resonance between the electrostatic coupling of the line and the compensating shunt reactors. Engineers of the Texas Electric Service (TES) Company [1] and the General Electric Company [2] have reported their findings and have recommended several corrective actions. One of the recommendations was to transpose the lines, but since the TES circuit was already in operation, the calculation for transposition was not discussed. Therefore, this paper presents a method for determining the effects of transposition. Fortunately, the method has proven flexible so that the results include the effects of electromagnetic induction, electrostatic coupling, and the distributed lines.

The research for this paper was begun as part of a summer project for the Southern California Edison Company. The manipulation of the equations requires the use of a large digital computer. The initial work was conducted on the Southern California Edison's CDC 3400 computer, and completed on the Brigham Young University's IBM 7040 computer.

The equations in this paper have been written in matrix and simplified tensor forms. The approach employed has been to cascade the impedances and admittance of the $n$-dimensional arrays to determine a total system admittance. The total current can then be determined for a given sending-end voltage. Finally, the voltages and currents may be calculated for each section of the system.

This paper contains a description of the cascading equations, a brief description of the computer program, and a summary of the results. The Appendixes include the equations used in calculating the electromagnetic and electrostatic effects and the data for a sample case.

Paper 68 TP 617-PWR, recommended and approved by the Transmission and Distribution Committee of the IEEE Power Group for presentation at the IEEE Summer Power Meeting, Chicago, Ill., June 23-28, 1968. Manuscript submitted February 12, 1968; made available for printing April 2, 1968.

The author is with Brigham Young University, Provo, Utah.

\section{Matrix Equations}

The cascading of networks represented by the "generalized $A B C D$ constants" has been used by utility and electronic engineers for some time. Kron [3] and Bewley [4] have employed the more general $n$-dimensional arrays to represent electrical systems and have suggested ways to cascade subnetwork equations to form the equations for more complex systems. The next few paragraphs introduce the notation used in this paper.

The equations of a given subnetwork may be represented by a set of simultaneous equations, such as

$$
\begin{aligned}
& v_{1}=z_{11} i_{1}+z_{12} i_{2}+\cdots+z_{1 n} i_{n} \\
& v_{2}=z_{21} i_{1}+z_{22} i_{2}+\cdots+z_{2 n} i_{n} \\
& \begin{array}{llll}
\bullet & \bullet & \bullet & \bullet
\end{array} \\
& \text { - } .0 . \\
& v_{n}=z_{m 1} i_{1}+z_{m 2} i_{2}+\cdots+z_{m n} i_{n}
\end{aligned}
$$

where

$v_{n} \quad$ loop voltages

$z_{m n} \quad$ self-impedances when $m=n$

$z_{m n}$ mutual impedances when $m \neq n$.

The simultaneous equations (1) may be represented in the matrix form of (2) or in the simplified tensor form of (3).

$$
\left[\begin{array}{c}
v_{1} \\
v_{2} \\
\dot{\bullet} \\
\dot{v_{n}}
\end{array}\right]=\left[\begin{array}{cccc}
z_{11} & z_{12} & \cdots & z_{1 n} \\
z_{21} & z_{22} & \cdots & z_{2 n} \\
\cdot & \cdot & & \cdot \\
\cdot & \cdot & & \cdot \\
\cdot & \cdot & & \cdot \\
z_{m 1} & z_{m 2} & \cdots & z_{m n}
\end{array}\right]\left[\begin{array}{c}
i_{1} \\
i_{2} \\
\dot{\cdot} \\
i_{n}
\end{array}\right]
$$

The equations for a subnetwork can also be represented in the admittance matrix form of (4) or the tensor form of (5).

$$
\left[\begin{array}{c}
i_{1} \\
i_{2} \\
\cdot \\
\cdot \\
\cdot \\
i_{n}
\end{array}\right]=\left[\begin{array}{llll}
y_{11} & y_{12} & \cdots & y_{1 n} \\
y_{21} & y_{22} & \cdots & y_{2 n} \\
\cdot & \cdot & & \cdot \\
\cdot & \cdot & & \cdot \\
\cdot & \cdot & & \cdot \\
y_{m 1} & y_{m 2} & \cdots & y_{m n}
\end{array}\right]\left[\begin{array}{c}
v_{1} \\
v_{2} \\
\cdot \\
\cdot \\
v_{n}
\end{array}\right]
$$

where

$y_{m n} \quad$ self-admittances when $m=n$

$y_{m n}$ mutual admittances when $m \neq n$.

$$
I=Y V \text {. }
$$




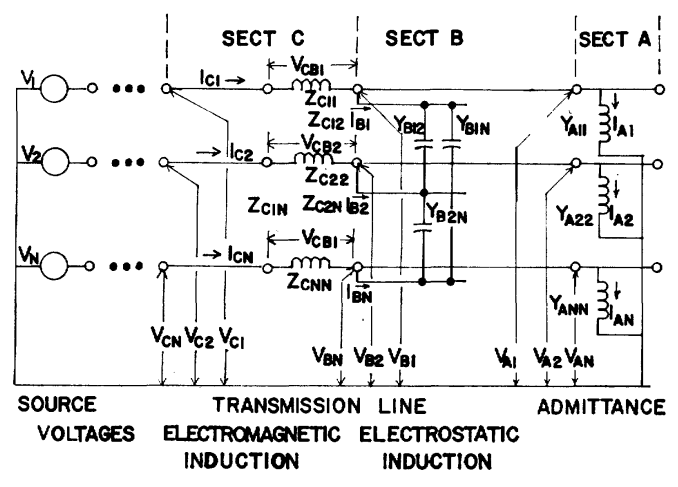

Fig. 1. Circuit diagram.

The impedance and admittance coefficient matrices are matrix inversions of each other, or

$$
Y=Z^{-1}
$$

\section{General Form of Network Matrix Equations}

The circuit diagram for a transmission line network is given in Fig. 1. The following is a development of a set of matrix equations which provide a method of calculating the voltages and currents in each section of the given transmission line system. Begin at the receiving end of the network and then solve for the impedances and admittances. At the receiving end

where

$$
V_{a}=Z_{a} I_{a}
$$

$V_{a}=\left[\begin{array}{l}V_{a 1} \\ V_{a 2} \\ \cdot \\ \cdot \\ V_{a n}\end{array}\right], Z_{a}=\left[\begin{array}{cccc}Z_{a 11} & 0 & \cdots & 0 \\ 0 & Z_{a 22} & \cdots & 0 \\ \cdot & \cdot & & \cdot \\ \cdot & \cdot & & \cdot \\ \cdot & \cdot & & \cdot \\ 0 & 0 & \cdots & Z_{a m n}\end{array}\right], \quad I_{a}=\left[\begin{array}{l}I_{a 1} \\ I_{a 2} \\ \cdot \\ \cdot \\ \dot{I_{a n}}\end{array}\right]$.

The inverse of $(7)$ is

$$
I_{a}=Z_{a}^{-1} V_{a}=Y_{a} V_{a}=Y_{a} V_{t} .
$$

For section $b$

$$
I_{b}=Y_{b} V_{b}
$$

where

$$
I_{b}=\left[\begin{array}{l}
I_{b 1} \\
I_{b 2} \\
\cdot \\
\dot{I_{b n}}
\end{array}\right], V_{b}=V_{a}=\left[\begin{array}{l}
V_{b 1} \\
V_{b 2} \\
\dot{\cdot} \\
\cdot \\
V_{b n}
\end{array}\right]
$$

and $Y_{b}$ is defined by (41) and (42).

Combine the currents of sections $a$ and $b$ to obtain the current of section $c$, so that

$$
\begin{aligned}
I_{c} & =I_{a}+I_{b}=Y_{a} V_{b}+Y_{b} V_{b}=\left(Y_{a}+Y_{b}\right) V_{b} \\
& =Y_{a b} V_{b} .
\end{aligned}
$$

The inverse of (12) is

$$
V_{b}=Y_{a b}{ }^{-1} I_{c}=Z_{a b} I_{c} .
$$

The voltages at the sending end of section $c$ are the sum of $V_{b}$ and the voltage of section $c$, thus

$$
\begin{aligned}
V_{c} & =V_{b}+Z_{c} I_{c}=Z_{a b} I_{c}+Z_{c} I_{c}=\left(Z_{a b}+Z_{c}\right) I_{c} \\
& =Z_{a b c} I_{c} .
\end{aligned}
$$

The impedance matrix $Z_{c}$ for the electromagnetic induction is given by (21).

Finally the source current may be found from the inverse of (14) or

$$
I_{c}=Z_{a b c}^{-1} V_{c}=Y_{a b c} V_{c}
$$

The voltages and currents of each section may be determined by reversing the direction of calculations, i.e.,

$$
\begin{aligned}
V_{c b} & =Z_{c} I_{c} \\
V_{b} & =V_{c}-V_{c b} \\
I_{b} & =Y_{b} V_{b} \\
I_{a} & =I_{c}-I_{b} \\
V_{a} & =V_{b} .
\end{aligned}
$$

Equations (7)-(20) outline the method of solution for a transmission system. More complex systems would probably contain more sections. Series capacitors, shunt reactors, and loads may be added as an impedance or admittance matrix as required. The distributed effect of the line is slightly more involved. Each transmission line consists of the electromagnetic and electrostatic features. The distributed effect is accounted for by repeating the electrostatic and electromagnetic sections as many times as desired. The results portrayed in Table II indicate that good accuracy can be obtained by dividing the line into divisions of $20-40$ miles. Shorter divisions could be employed at a sacrifice of increased computer time. The computer program has been written so that the number of divisions is determined by a simple code number on the control card. Transposition may be accomplished by changing the conductor configuration. The computer program was written to do this by a coding of the required data.

\section{Description of the Computer Program}

The computer program is written in Fortran IV. The data required includes the conductor resistance $r_{a}$, conductor radius $r$, tower configuration dimensions, length of transmission line, number of conductors, impedance of line elements, and the sending-end voltages.

The main program solves (7)-(20) and requires several subprograms. One subprogram calculates the transmission line electromagnetic and electrostatic coefficients from the given data. The remaining subprograms perform the matrix manipulations of multiplication, inversion, addition, subtraction, and multiplication by a constant. All the manipulations are performed with complex numbers. The computer printout can be represented in an abbreviated form or can be made to include the details of each major calculation. 
TABLE I

Summary of Voltages and Currents for 200 Miles of 500-kV Parallel Lines with the Transmission Line Divided in Two Divisions and 125-Mvar Shunt Reactors at Each End*

\begin{tabular}{|c|c|c|c|c|c|c|c|c|c|c|c|c|}
\hline & \multicolumn{12}{|c|}{ Conductors $\dagger$} \\
\hline & \multicolumn{2}{|c|}{1} & \multicolumn{2}{|c|}{2} & \multicolumn{2}{|c|}{3} & \multicolumn{2}{|c|}{4} & \multicolumn{2}{|c|}{5} & \multicolumn{2}{|c|}{6} \\
\hline & $A$ & $\angle a^{\circ}$ & $A$ & $\angle a^{\circ}$ & $A$ & $\angle a^{\circ}$ & $A$ & $\angle a^{\circ}$ & $A$ & $\angle a^{\circ}$ & $A$ & $\angle a^{\circ}$ \\
\hline \multicolumn{13}{|l|}{ Sending end } \\
\hline $\begin{array}{l}\text { Voltage } V_{4} \\
\text { Current } I_{43}\end{array}$ & $\begin{array}{l}303 \\
513\end{array}$ & $\begin{array}{r}0 \\
99\end{array}$ & $\begin{array}{l}303 \\
432\end{array}$ & $\begin{array}{r}-120 \\
-25\end{array}$ & $\begin{array}{l}303 \\
560\end{array}$ & $\begin{array}{l}120 \\
177\end{array}$ & & & & & & \\
\hline \multicolumn{13}{|c|}{ Sending-end reactor } \\
\hline $\begin{array}{l}\text { Voltage } V_{3} \\
\text { Current } I_{4}\end{array}$ & & & & & & & $\begin{array}{r}1530 \\
694\end{array}$ & $\begin{array}{r}24 \\
-66\end{array}$ & $\begin{array}{l}185 \\
837\end{array}$ & $\begin{array}{r}26 \\
-65\end{array}$ & $\begin{array}{r}1564 \\
709\end{array}$ & $\begin{array}{l}26 \\
64\end{array}$ \\
\hline \multicolumn{13}{|c|}{ Sections 3 and 2} \\
\hline $\begin{array}{c}\text { Division } 2 \\
V_{32} \\
I_{32} \\
I_{2} \\
V_{2}\end{array}$ & $\begin{array}{r}30 \\
265 \\
249 \\
274\end{array}$ & $\begin{array}{r}13 \\
99 \\
99 \\
-1\end{array}$ & $\begin{array}{r}80 \\
219 \\
213 \\
370\end{array}$ & $\begin{array}{r}22 \\
-24 \\
-25 \\
-128\end{array}$ & $\begin{array}{r}75 \\
287 \\
273 \\
360\end{array}$ & $\begin{array}{r}-15 \\
176 \\
177 \\
128\end{array}$ & $\begin{array}{r}14 \\
31 \\
724 \\
1670\end{array}$ & $\begin{array}{r}15 \\
105 \\
-66 \\
-156\end{array}$ & $\begin{array}{r}155 \\
34 \\
871 \\
2000\end{array}$ & $\begin{array}{r}17 \\
107 \\
-65 \\
-155\end{array}$ & $\begin{array}{r}143 \\
32 \\
741 \\
1710\end{array}$ & $\begin{array}{r}17 \\
107 \\
-64 \\
-155\end{array}$ \\
\hline $\begin{array}{c}\text { Division } 1 \\
V_{21} \\
I_{21} \\
I_{2} \\
V_{1}\end{array}$ & $\begin{array}{r}30 \\
0 \\
265 \\
304\end{array}$ & $\begin{array}{r}-171 \\
99 \\
0\end{array}$ & $\begin{array}{r}11 \\
0 \\
219 \\
368\end{array}$ & $\begin{array}{r}149 \\
-24 \\
-126\end{array}$ & $\begin{array}{r}26 \\
0 \\
29 \\
368\end{array}$ & $\begin{array}{r}-126 \\
176 \\
124\end{array}$ & $\begin{array}{r}16 \\
749 \\
719 \\
1650\end{array}$ & $\begin{array}{r}-160 \\
114 \\
-66 \\
-156\end{array}$ & $\begin{array}{r}16 \\
900 \\
866 \\
1980\end{array}$ & $\begin{array}{r}-162 \\
115 \\
-65 \\
-155\end{array}$ & $\begin{array}{r}15 \\
767 \\
735 \\
1690\end{array}$ & $\begin{array}{r}-163 \\
115 \\
-64 \\
-155\end{array}$ \\
\hline \multicolumn{13}{|c|}{ Receiving-end reactor } \\
\hline $\begin{array}{l}\text { Voltage } V_{1} \\
\text { Current } I_{1}\end{array}$ & & & & & & & $\begin{array}{r}1650 \\
749\end{array}$ & $\begin{array}{r}-156 \\
114\end{array}$ & $\begin{array}{r}1980 \\
900\end{array}$ & $\begin{array}{r}-155 \\
115\end{array}$ & $\begin{array}{r}1690 \\
767\end{array}$ & $\begin{array}{r}-155 \\
115\end{array}$ \\
\hline
\end{tabular}

* See Figs. 2(b) and 5.

$\dagger$ See Fig. 5.

TABLE II

Distributed Effect of a 200-Mile Line

\begin{tabular}{|c|c|c|c|c|c|c|c|c|c|c|c|c|c|}
\hline \multirow{2}{*}{\multicolumn{2}{|c|}{$\begin{array}{l}\text { Number of Divisions } \\
\text { Miles per Division }\end{array}$}} & \multirow{2}{*}{\multicolumn{2}{|c|}{$\begin{array}{c}1 \\
200\end{array}$}} & \multirow{2}{*}{\multicolumn{2}{|c|}{$\begin{array}{c}2 \\
100\end{array}$}} & \multirow{2}{*}{\multicolumn{2}{|c|}{$\begin{array}{r}5 \\
40\end{array}$}} & \multirow{2}{*}{\multicolumn{2}{|c|}{$\begin{array}{l}10 \\
20\end{array}$}} & \multirow{2}{*}{\multicolumn{2}{|c|}{$\begin{array}{l}20 \\
10\end{array}$}} & \multirow{2}{*}{\multicolumn{2}{|c|}{$\begin{array}{r}50 \\
4\end{array}$}} \\
\hline & & & & & & & & & & & & & \\
\hline Conductor & Mvars & $A$ & $\angle a^{\circ}$ & $A$ & $\angle a^{\circ}$ & $A$ & $\angle a^{\circ}$ & $A$ & $\angle a^{\circ}$ & $A$ & $\angle a^{\circ}$ & $A$ & $\angle a^{\circ}$ \\
\hline \multicolumn{14}{|c|}{ Sending-End Currents (A) } \\
\hline $\begin{array}{l}1 \\
2 \\
3 \\
4 \\
5 \\
6\end{array}$ & 50 & $\begin{array}{r}519 \\
522 \\
515 \\
5 \\
3 \\
2\end{array}$ & $\begin{array}{r}95 \\
-30 \\
-156 \\
-132 \\
-126 \\
-117\end{array}$ & $\begin{array}{r}481 \\
515 \\
478 \\
5 \\
3 \\
2\end{array}$ & $\begin{array}{r}95 \\
-30 \\
-156 \\
-135 \\
-132 \\
-129\end{array}$ & $\begin{array}{r}464 \\
498 \\
461 \\
6 \\
3 \\
2\end{array}$ & $\begin{array}{r}95 \\
-30 \\
-156 \\
-136 \\
-134 \\
-133\end{array}$ & $\begin{array}{r}459 \\
494 \\
456 \\
6 \\
3 \\
2\end{array}$ & $\begin{array}{r}95 \\
-30 \\
-156 \\
-136 \\
-135 \\
-133\end{array}$ & $\begin{array}{r}457 \\
491 \\
454 \\
6 \\
3 \\
2\end{array}$ & $\begin{array}{r}95 \\
-30 \\
-156 \\
-137 \\
-135 \\
-133\end{array}$ & $\begin{array}{r}455 \\
490 \\
453 \\
6 \\
3 \\
2\end{array}$ & $\begin{array}{r}95 \\
-30 \\
-156 \\
-137 \\
-135 \\
-134\end{array}$ \\
\hline $\begin{array}{l}1 \\
2 \\
3 \\
4 \\
5 \\
6\end{array}$ & 125 & $\begin{array}{l}518 \\
545 \\
490 \\
128 \\
141 \\
113\end{array}$ & $\begin{array}{r}96 \\
-30 \\
-159 \\
-117 \\
-115 \\
-114\end{array}$ & $\begin{array}{l}513 \\
432 \\
560 \\
694 \\
837 \\
710\end{array}$ & $\begin{array}{r}99 \\
-25 \\
177 \\
-66 \\
-65 \\
-64\end{array}$ & $\begin{array}{l}469 \\
415 \\
688 \\
601 \\
741 \\
637\end{array}$ & $\begin{array}{r}102 \\
-37 \\
-165 \\
-4 \\
-3 \\
-3\end{array}$ & $\begin{array}{l}464 \\
413 \\
678 \\
571 \\
706 \\
608\end{array}$ & $\begin{array}{r}103 \\
-37 \\
-164 \\
-1 \\
0 \\
0\end{array}$ & $\begin{array}{l}463 \\
409 \\
677 \\
572 \\
707 \\
609\end{array}$ & $\begin{array}{r}103 \\
-38 \\
-164 \\
-2 \\
-1 \\
0\end{array}$ & $\begin{array}{l}463 \\
405 \\
677 \\
598 \\
714 \\
615\end{array}$ & $\begin{array}{r}103 \\
-37 \\
-164 \\
-2 \\
-1 \\
-1\end{array}$ \\
\hline $\begin{array}{l}1 \\
2 \\
3 \\
4 \\
5 \\
6\end{array}$ & 200 & $\begin{array}{r}518 \\
550 \\
535 \\
116 \\
40 \\
107\end{array}$ & $\begin{array}{r}95 \\
-31 \\
-156 \\
36 \\
41 \\
-144\end{array}$ & $\begin{array}{r}479 \\
513 \\
493 \\
78 \\
43 \\
76\end{array}$ & $\begin{array}{r}96 \\
-31 \\
-156 \\
38 \\
42 \\
-141\end{array}$ & $\begin{array}{r}462 \\
497 \\
476 \\
72 \\
45 \\
70\end{array}$ & $\begin{array}{r}96 \\
-31 \\
-155 \\
38 \\
42 \\
-140\end{array}$ & $\begin{array}{r}457 \\
492 \\
471 \\
70 \\
45 \\
70\end{array}$ & $\begin{array}{r}96 \\
-31 \\
-155 \\
38 \\
42 \\
-140\end{array}$ & $\begin{array}{r}455 \\
490 \\
496 \\
70 \\
45 \\
69\end{array}$ & $\begin{array}{r}96 \\
-31 \\
-155 \\
39 \\
42 \\
-140\end{array}$ & $\begin{array}{r}453 \\
488 \\
468 \\
70 \\
45 \\
69\end{array}$ & $\begin{array}{r}96 \\
-31 \\
-155 \\
31 \\
42 \\
-140\end{array}$ \\
\hline \multicolumn{14}{|c|}{ Sending-End Reactor Voltages $(\mathrm{kV})$} \\
\hline $\begin{array}{l}4 \\
5 \\
6\end{array}$ & 50 & $\begin{array}{r}28 \\
16 \\
9\end{array}$ & $\begin{array}{l}-42 \\
-35 \\
-27\end{array}$ & $\begin{array}{l}30 \\
18 \\
11\end{array}$ & $\begin{array}{l}-45 \\
-42 \\
-39\end{array}$ & $\begin{array}{l}30 \\
19 \\
12\end{array}$ & $\begin{array}{l}-46 \\
-45 \\
-43\end{array}$ & $\begin{array}{l}31 \\
19 \\
12\end{array}$ & $\begin{array}{l}-47 \\
-45 \\
-43\end{array}$ & $\begin{array}{l}31 \\
19 \\
12\end{array}$ & $\begin{array}{l}-47 \\
-45 \\
-44\end{array}$ & $\begin{array}{l}31 \\
19 \\
12\end{array}$ & $\begin{array}{l}-47 \\
-46 \\
-44\end{array}$ \\
\hline $\begin{array}{l}4 \\
5 \\
6\end{array}$ & 125 & $\begin{array}{l}283 \\
311 \\
249\end{array}$ & $\begin{array}{l}-28 \\
-25 \\
-24\end{array}$ & $\begin{array}{l}1530 \\
1846 \\
1564\end{array}$ & $\begin{array}{l}24 \\
25 \\
26\end{array}$ & $\begin{array}{l}1324 \\
1634 \\
1404\end{array}$ & $\begin{array}{l}86 \\
87 \\
87\end{array}$ & $\begin{array}{l}1259 \\
1556 \\
1340\end{array}$ & $\begin{array}{l}89 \\
90 \\
90\end{array}$ & $\begin{array}{l}1262 \\
1560 \\
1344\end{array}$ & $\begin{array}{l}88 \\
89 \\
90\end{array}$ & $\begin{array}{l}1274 \\
1575 \\
1357\end{array}$ & $\begin{array}{l}88 \\
89 \\
89\end{array}$ \\
\hline $\begin{array}{l}4 \\
5 \\
6\end{array}$ & 200 & $\begin{array}{r}160 \\
55 \\
148\end{array}$ & $\begin{array}{r}126 \\
133 \\
-54\end{array}$ & $\begin{array}{r}108 \\
59 \\
104\end{array}$ & $\begin{array}{r}128 \\
132 \\
-51\end{array}$ & $\begin{array}{l}99 \\
61 \\
97\end{array}$ & $\begin{array}{r}128 \\
132 \\
-51\end{array}$ & $\begin{array}{l}97 \\
62 \\
96\end{array}$ & $\begin{array}{r}128 \\
132 \\
-50\end{array}$ & $\begin{array}{l}97 \\
62 \\
96\end{array}$ & $\begin{array}{r}128 \\
132 \\
-50\end{array}$ & $\begin{array}{l}97 \\
62 \\
96\end{array}$ & $\begin{array}{r}128 \\
132 \\
-50\end{array}$ \\
\hline
\end{tabular}




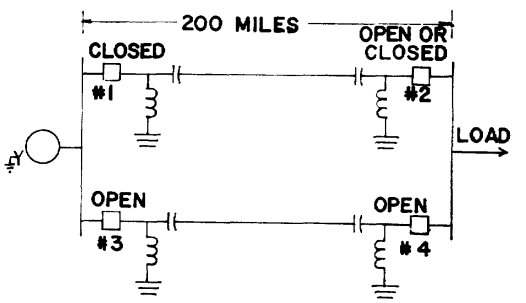

(a)

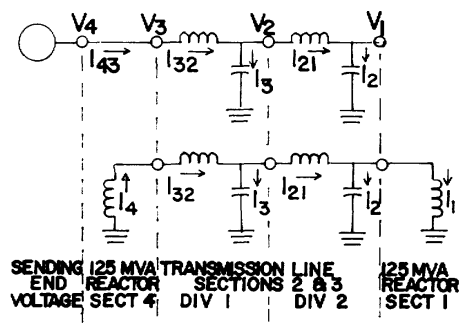

(b)

Fig. 2. (a) One line diagram for example problem. (b) One line impedance diagram for calculation of Table I.

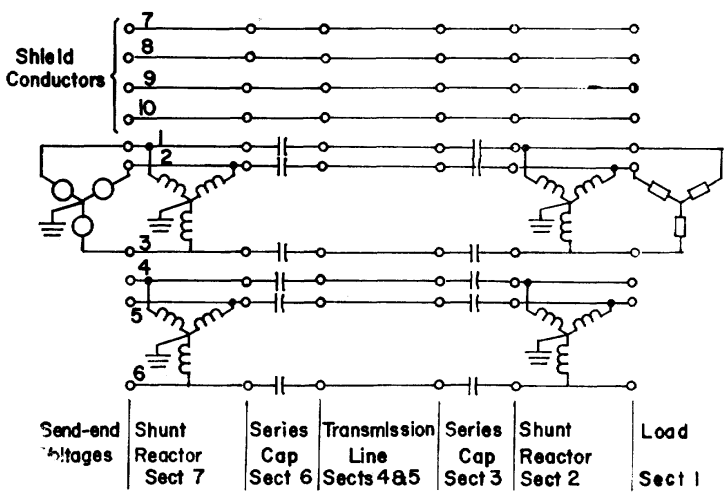

Fig. 3. Circuit diagram for example problem.

\section{Results of Calculations}

Several examples of application are included as Appendix III. The following is a summary of results. It must be remembered that all these results are based on a linear shunt reactor and that the effect of reactor nonlinearity has not been included.

\section{System Voltage and Currents}

The section voltages and currents for the system of Figs. 2-5 are included in Table I. The transmission line may be divided into more divisions to increase the accuracy as has been done for most of the other parts of the example.

\section{Effects of Distributed Line}

A 200-mile line of Fig. 2(a) has been divided into 1-50 divisions (200-4 miles, respectively) with the results included in Table II. The 40-mile division has been selected for the majority of calculations as a compromise between accuracy and computing time.

\section{Various Values of Shunt Reactors}

Fig. 6(a) is a plot of the reactor voltages for various values of reactor rating in Mvars. It will be noted that there are several resonance values, a condition which would be expected because of the various values of capacitance between the respective conductors. Fig. 6(b) and (c) have been added to clarify the peak values near resonance. (The format of Fig. 6 may be compared with Hesse's results [2].)

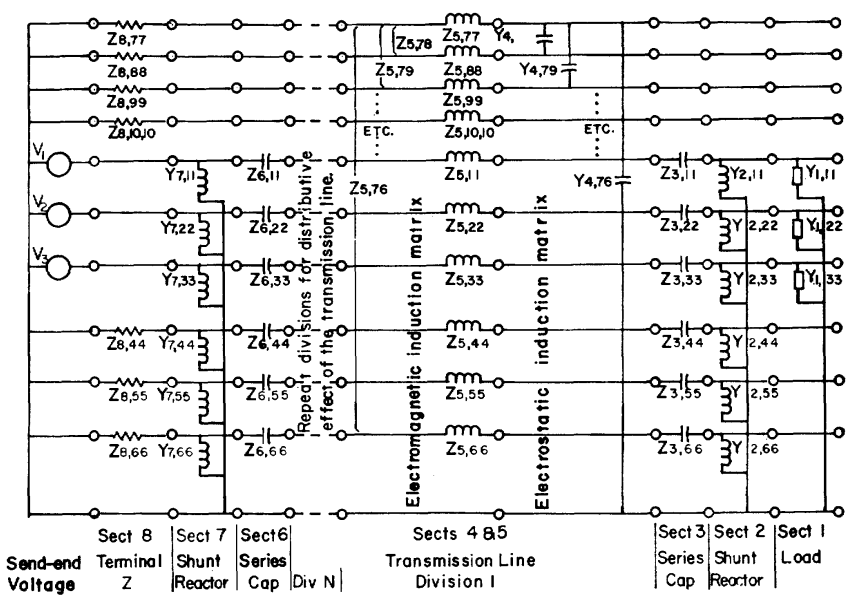

Fig. 4. Circuit diagram for example problem adapted for the digital computer program.

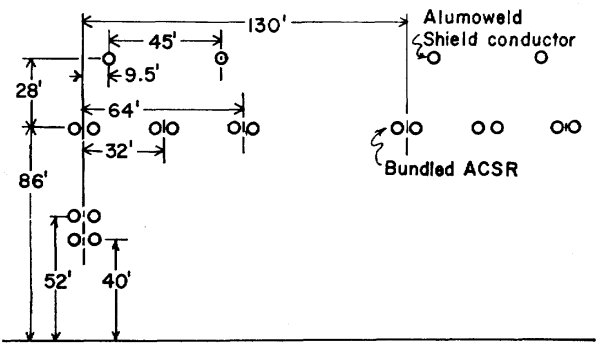

Fig. 5. Two-circuit 500-kV transmission tower configuration.

\section{Transposition}

Two schemes of transposition were considered for review. Scheme 1 involves transposing the conductors within each circuit only as shown in Fig. 7, whereas scheme 2 involves transposing all conductors as shown in Fig. 8. Plots of voltage versus reactor Mvars for the two transposition schemes have been prepared as Figs. 9 and 10, respectively. It is obvious that the transposition does reduce the magnitude of the induced voltages by almost four in scheme 1 and by seven in scheme 2 for smaller Mvar ratings of the reactors. Near resonance, though, the high induced voltage problem still exists. Care must be used when considering transposition as the solution, particularly if the line is to be divided into smaller sections at a subsequent time. 


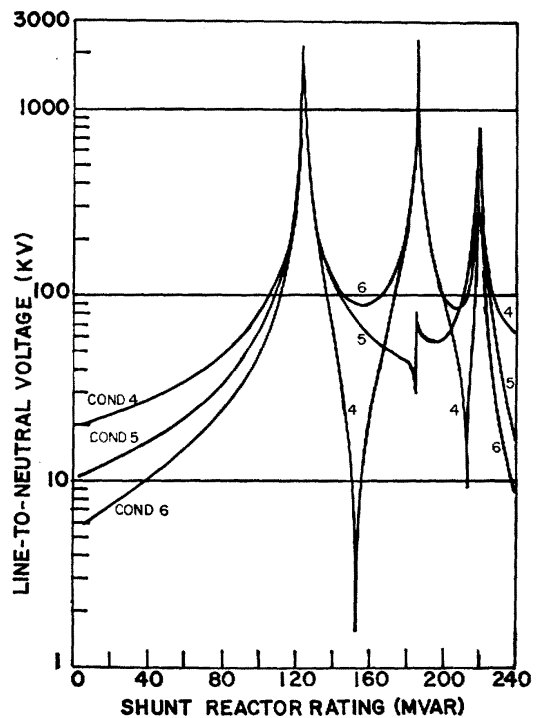

(a)

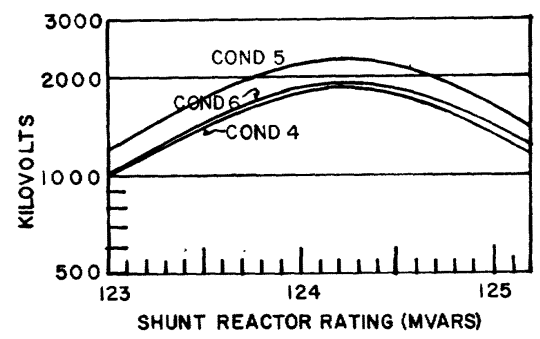

(b)

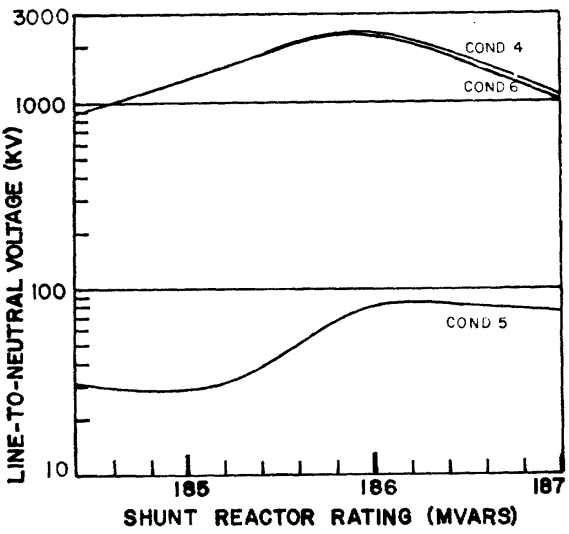

(c)

Fig. 6. (a) Sending-end shunt reactor voltage for the 200-mile line of Fig. 2(b) for various reactor ratings. (b), (c) Expanded scales for sections of (a).

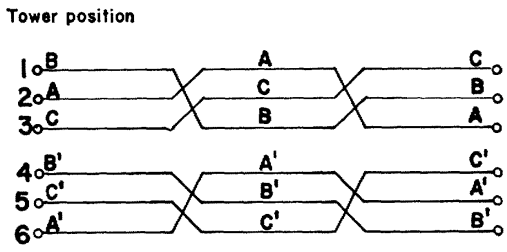

Fig. 7. Transposition scheme 1 (see Fig. 5).

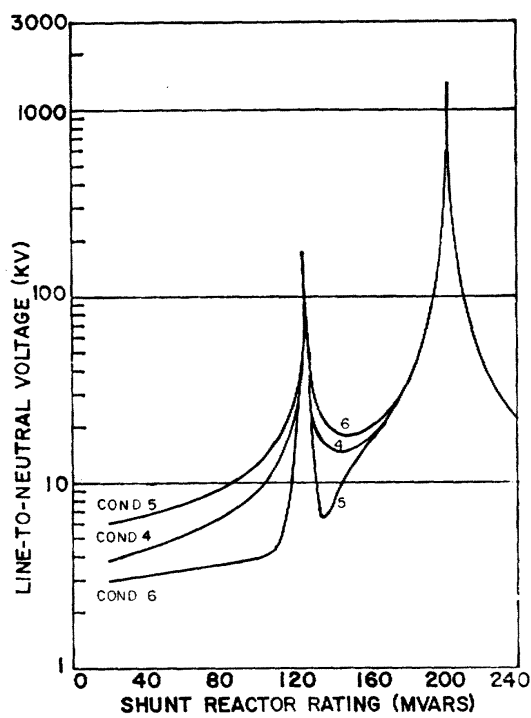

Fig. 9. Sending-end shunt reactor voltage for a 200-mile line with transposition scheme 1 (Fig. 7)

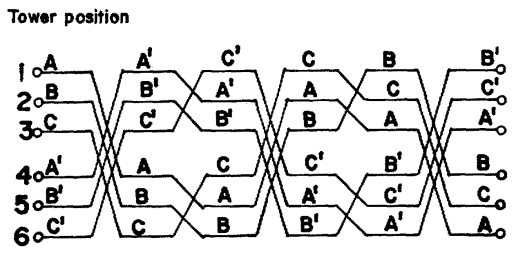

Fig. 8. Transposition scheme 2 (see Fig. 5).

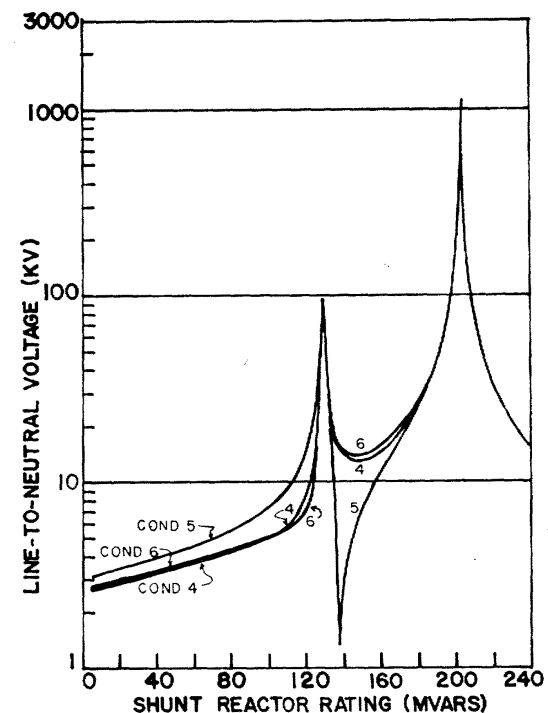

Fig. 10. Sending-end shunt reactor voltage for a 200-mile line with transposition scheme 2 (Fig. 8). 


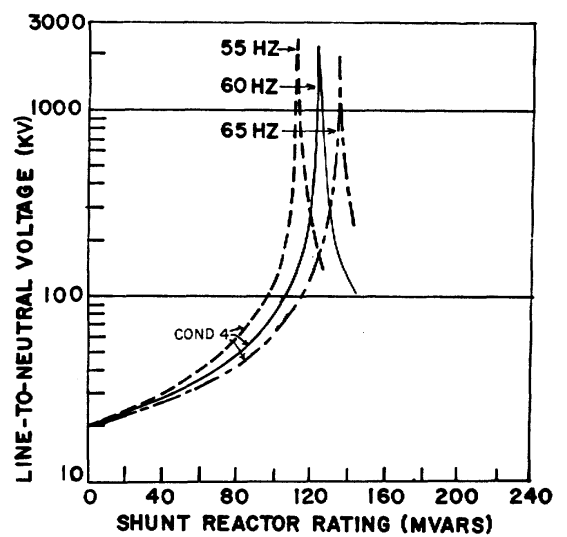

Fig. 11. Sending-end shunt reactor voltage for the 200-mile line of Fig. 2(b) at various frequencies.

\section{Various Frequencies}

Fig. 11 is a plot of voltage versus reactor Mvars for various values of frequency. The effect of frequency change should be obvious from the plots.

\section{Conclusion}

The method of calculating transmission line parameters as described in this paper provides a method for determining the steady-state features of a transm ssion system. It is shown that transposition is one method for reducing the effects of electrostatic resonance for the lower values of shunt reactor Mvars. Several calculations have been included, but many other features could be considered, such as calculating optimum location of series capacitors and shunt reactors, optimum spacing of conductors, and optimum transposition schemes.

\section{APPENDIX I}

\section{Electromagnetic Coefficients}

\section{Carson Equations}

The computer program calculates the electromagnetic coefficients by solving the equations developed by Carson [5]. Clarke [6] added several terms and Calabrese [7] has modernized some of the constants. A summary of the equations follows. In matrix form these equations are

$$
\left[\begin{array}{l}
V_{1} \\
V_{2} \\
\cdot \\
\cdot \\
V_{n}
\end{array}\right]=\left[\begin{array}{llll}
Z_{11} & Z_{12} & \cdots & Z_{1 n} \\
Z_{21} & Z_{22} & \cdots & Z_{2 n} \\
\cdot & \cdot & & \cdot \\
\cdot & \cdot & & \cdot \\
Z_{m 1} & Z_{m 2} & \cdots & Z_{m n}
\end{array}\right]\left[\begin{array}{l}
I_{1} \\
I_{2} \\
\cdot \\
\cdot \\
I_{n}
\end{array}\right] .
$$

In modified tensor form (21) is

$$
V=Z I \text {. }
$$

When $m=n$

$$
\begin{array}{rl}
Z_{m n}=R_{m}+j 0.004 & 657 f \log \left(D_{m n^{\prime}} / G M R_{m}\right) \\
+ & 0.004043 f(P+j Q) \text { ohms } / \mathrm{mile}
\end{array}
$$

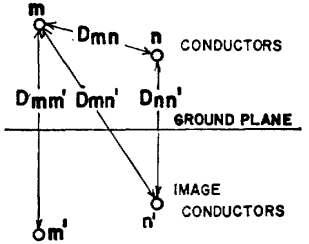

Fig. 12. Tower spacing dimension symbols.

When $m \neq n$

$Z_{m n}=j 0.004657 f \log \left(D_{m n^{\prime}} / D_{m n}\right)$

$$
+0.004043 f(P+j Q) \text { ohms } / \text { mile }
$$

where

$$
\begin{array}{ll}
f & \text { frequency, hertz } \\
\mathrm{GMR}_{m} & \text { geometric mean radius for conductor } m, \text { feet } \\
D_{m n} & \text { distance between conductors } m \text { and } n \text {, feet } \\
D_{m m^{\prime}}, D_{m n^{\prime}} & \begin{array}{l}
\text { distance between conductors } m \text { and image con- } \\
\text { ductor } m^{\prime} \text { or } n^{\prime} \text { (see Fig. } 12 \text { ), feet }
\end{array} \\
R_{m} & \text { ac resistance of conductor } m, \text { ohms } / \text { mile. }
\end{array}
$$

The terms $P$ and $Q$ are defined by the following expression:

$$
\begin{gathered}
P=\frac{\pi}{8}-\frac{1}{3 \sqrt{2}} k \cos \theta+\frac{k^{2}}{16} \cos 2 \theta\left(0.6728+\ln \frac{2}{k}\right) \\
+\frac{k^{2}}{16} \theta \sin 2 \theta+\frac{k^{3} \cos 3 \theta}{45 \sqrt{2}}-\frac{\pi k^{4} \cos 4 \theta}{1536} \\
Q=-0.0386+\frac{1}{2} \ln \frac{2}{k}+\frac{1}{3 \sqrt{2}} k \cos \theta-\frac{\pi k}{64} \cos 2 \theta+\frac{k^{3} \cos 3 \theta}{45 \sqrt{2}} \\
-\frac{k^{4} \theta}{384} \sin 4 \theta-\frac{k^{4} \cos 4 \theta}{384}\left(\ln \frac{2}{k}+1.0895\right)
\end{gathered}
$$

The terms $P$ and $Q$ have different values for the impedance coefficients $Z_{m n}$ when $m=n$ and $m \neq n$.

In the case of $m=n$

$$
\begin{aligned}
& k=2.80 \times 10^{-3} D_{m m^{\prime}} \sqrt{f / \rho} \\
& \theta=0 .
\end{aligned}
$$

In the case of $m \neq n$

$$
\begin{aligned}
& k=2.80 \times 10^{-3} D_{m m^{\prime}} \sqrt{f / \rho} \\
& \theta=\arcsin \left(H_{m n} / D_{m n^{\prime}}\right)
\end{aligned}
$$

where

$f \quad$ frequency, hertz

$\rho$ resistivity of earth, ohms/meter

$H_{m n}$ horizontal distance between conductors $m$ and $n$

$D_{m n}$, image distance as shown in Fig. 12.

\section{Approximating Equations}

Most electromagnetic calculations have been made with the approximating equations listed below [8], [9]; therefore, the computer program can be coded to use these equations whenever the operator feels it desirable. The impedance value at $60 \mathrm{~Hz}$ 
for $m=n$ is

$$
Z_{m n}=Z_{m m^{\prime}}+Z_{g}-2 Z_{m g} \mathrm{ohms} / \mathrm{mile}
$$

and $m \neq n$ is

$$
Z_{m n}=Z_{m n^{\prime}}+Z_{g}-Z_{m g}-Z_{n g} \text { ohms } / \text { mile }
$$

where

$$
\begin{aligned}
& Z_{m m^{\prime}} \quad \text { self-impedance of conductor } \\
& r_{m}+j 0.27940 \log \left(1 / \mathrm{GMR}_{m}\right) \\
& Z_{m n^{\prime}} \quad \text { mutual impedance between conductors } \\
& j 0.27940 \log \left(1 / D_{m n}\right) \\
& Z_{g} \quad \text { self-impedance of ground circuit } \\
& 0.09528+j 0 \\
& Z_{m g} \quad Z_{n g}=\text { mutual impedance between conductor and } \\
& \text { ground }=j 0.1397 \log (1 / 2160 \sqrt{ } \rho / f) \\
& r_{m} \quad \text { resistance of conductor, ohms } / \mathrm{mile} \\
& D_{m n} \quad \text { distance between conductors, feet } \\
& \mathrm{GMR}_{m} \text { geometric mean radius of conductor } m \text {, feet } \\
& \rho \text { resistivity, ohms/meter } \\
& f \quad \text { frequency, hertz. }
\end{aligned}
$$

\section{Appendix II}

\section{Electrostatic Coefficients}

The equations for electrostatic induction in terms of voltages and electric charges are $[6]-[8]$

$$
\left[\begin{array}{c}
v_{1} \\
v_{2} \\
\cdot \\
\cdot \\
v_{n}
\end{array}\right]=\left[\begin{array}{llll}
P_{11} & P_{12} & \cdots & P_{1 n} \\
P_{21} & P_{22} & \cdots & P_{2 n} \\
\cdot & \cdot & & \cdot \\
\cdot & \cdot & & \cdot \\
\cdot & \cdot & & \cdot \\
P_{m 1} & P_{m 2} & \cdots & P_{m n}
\end{array}\right]\left[\begin{array}{l}
q_{1} \\
q_{2} \\
\cdot \\
\cdot \\
q_{n}
\end{array}\right]
$$

where $q$ are the conductor charges in coulombs, $v$ the conductor potentials in volts with respect to ground, and $P$ the "potential coefficients" defined by (34) and (35).

When $m=n$

$$
P_{m n}=2.5703 \times 10^{7} \log \left(D_{m n^{\prime}} / r_{m}\right) \text { daraf-miles. }
$$

When $m \neq n$

$$
P_{m n}=2.5703 \times 10^{7} \log \left(D_{m n^{\prime}} / D_{m n}\right) \text { daraf-miles }
$$

where

$$
\begin{array}{ll}
r_{m} & \text { radius of each conductor } \\
D_{m n} & \text { distance between conductors } m \text { and } n \\
D_{m n^{\prime}} & \text { distance between conductor } m \text { and the image conductor } \\
& n^{\prime} \text { (see Fig. 12). }
\end{array}
$$

The tensor form of (33) is

$$
V=P Q \text {. }
$$

In order to calculate the current flow, it is necessary to write (33) and (36) in terms of currents rather than electric charges; therefore, premultiply each side of $(36)$ by the inverse of $P$

$$
P^{-1} V=P^{-1} P Q=Q
$$

or

$$
Q=P^{-1} V
$$

For sinusoidal charges

$$
q_{m}(t)=Q_{m(\max )} \sin \omega t .
$$

Since $i=d q / d t$

$$
\begin{aligned}
i_{m}(t) & =\omega Q_{m(\max )} \cos \omega t \\
& =\omega Q_{m(\max )} \sin \left(\omega t+90^{\circ}\right) \\
& =j \omega Q_{m(\max )} \sin \omega t .
\end{aligned}
$$

The charging current of (39) is written in phasor form

$$
\dot{I}_{m}=j \omega \dot{Q}_{m(\max )} \text {. }
$$

Combine (40) with (37), thus

$$
I=j \omega Q=j \omega P^{-1} V=j \omega C V=Y V .
$$

The $C$ matrix is the inverse of the $P$ matrix where the $C$ matrix is referred to as the capacitance coefficients. In the expanded form (41) is

$$
\left[\begin{array}{l}
I_{1} \\
I_{2} \\
\cdot \\
\cdot \\
I_{n}
\end{array}\right]=j \omega\left[\begin{array}{llll}
C_{11} & C_{12} & \cdots & C_{1 n} \\
C_{21} & C_{22} & \cdots & C_{2 n} \\
\cdot & \cdot & & \cdot \\
\cdot & \cdot & & \cdot \\
\cdot & \cdot & & \cdot \\
C_{m 1} & C_{m 2} & \cdots & C_{m n}
\end{array}\right]\left[\begin{array}{l}
V_{1} \\
V_{2} \\
\cdot \\
\cdot \\
V_{n}
\end{array}\right] \cdot
$$

The inverse of (41) gives the potential coefficients in terms of the charging currents. Thus, by premultiplying with the inverse of $j \omega P^{-1}$

$$
V=(1 / j \omega) P I=Z I .
$$

In expanded form (43) is

$$
\left[\begin{array}{l}
V_{1} \\
V_{2} \\
\cdot \\
\cdot \\
V_{n}
\end{array}\right]=\frac{1}{j \omega}\left[\begin{array}{llll}
P_{11} & P_{12} & \cdots & P_{1 n} \\
P_{21} & P_{22} & \cdots & P_{2 n} \\
\cdot & \cdot & & \cdot \\
\cdot & \cdot & & \cdot \\
\cdot & \cdot & & \cdot \\
P_{m 1} & P_{m 2} & \cdots & P_{m m}
\end{array}\right]\left[\begin{array}{l}
I_{1} \\
I_{2} \\
\cdot \\
\cdot \\
I_{n}
\end{array}\right] .
$$

Appendix III

\section{Numerical Example}

Given the 200-mile long parallel $500-\mathrm{kV}$ transmission circuits of Figs. 2-5 inclusive, include capacitive compensation of 70 percent of the line inductive reactance with half the capacitance at each end of the line, and 125-Mvar shunt inductive compensation at the end of each circuit. The regular electrical loading is 1000 MVA per circuit.

\section{Main Conductor}

Bundled Bluebird ACSR with 18-inch spacing.

Single conductor data: $2156 \mathrm{kcmil}, 84 / 19$ strands, 1.762 -inch diameter, 0.05885 -foot geometric mean radius, 0.482 ac ohms per mile at $25^{\circ} \mathrm{C}$.

Bundled conductor data: $4312 \mathrm{kcmil}$

equivalent radius

$=\sqrt[4]{(\text { conductor radius })^{2}(\text { spacing })^{2}}$

$=\sqrt{(0.881)(18)}$

$=3.98$ inches, 


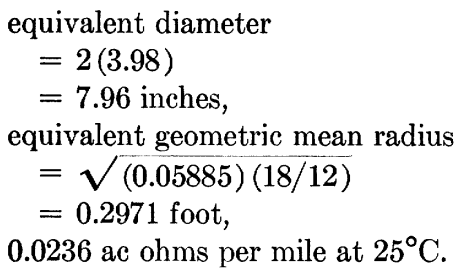

\section{Shield Conductor}

Seven strands of \# 6 AWG Alumoweld

0.486-inch diameter,

0.002633 -foot geometric mean radius,

$1.507 \mathrm{dc}$ ohms per mile at $25^{\circ} \mathrm{C}$.

\section{Shunt Reactors (wye connected)}

125 MVA at each end of each circuit reactance per phase

$$
=(\text { line voltage })^{2} / \mathrm{MVA}
$$$$
=(525)^{2} / 125
$$$$
=2205 \text { ohms per phase }
$$

resistance per phase for a $Q$ of 400 (where $Q$ is defined as $\omega L / R$ ) $R=X_{L} / Q=2205 / 400=5.51$ ohms per phase.

\section{Series Capacitors}

$$
\begin{aligned}
X_{c} & =70 \text { percent of } 0.5958-\text { line inductive reactance per mile } \\
& =-j 83.41 \mathrm{ohms} \text { for } 200 \text { miles of line } \\
& =-j 41.7 \mathrm{ohms} \text { at each end of each line. }
\end{aligned}
$$

\section{Problems}

The following are aspects of the numerical example which are summarized in the paper.

1) Evaluate the system voltages and currents when one circuit is de-energized and the other circuit is energized. Results: Table I.

2) Determine the sending-end reactor current and the sendingend shunt reactor voltage for the system of 1 ) for various values of shunt compensation. Results: Fig. 6 .

3) Solve 2) with the lines transposed as per Figs. 7 and 8. Results: Figs. 9 and 10.

4) Solve 2) with a various number of divisions of the transmission line section to evaluate the effects of the distributed line. Results: Table II.

5) Solve 2) for 55 and $65 \mathrm{~Hz}$. Results: Fig. 11.

\section{REFERENCES}

[1] M. J. Pickett, H. L. Manning, and N. Van Geem, "Near resonant coupling on EHV circuits: I-field investigations," IEEE Trans. Power Apparatus and Systems, vol. PAS-87, pp. 322325, February 1968

[2] M. H. Hesse and D. D. Wilson, "Near resonant coupling on EHV circuits: II-methods of analysis," IEEE Trans. Power Apparatus and Systems, vol. PAS-87, pp. 326-334, February 1968.

[3] G. Kron, Tensors for Circuits, 2nd ed. New York: Dover, 1959

[4] L. V. Bewley, Tensor Analysis of Electric Circuits and Machines. New York: Ronald, 1961.

[5] J. R. Carson, "Wave propagation in overhead wires with ground return," Bell Sys. Tech. J., vol. 5, pp. 539-554, 1926.

[6] E. Clarke, Circuit Analysis of A-C Power Systems, vol. 1. New York: Wiley, 1943, pp. 373-375, 434-442.

[7] G. O. Calabrese, Symmetrical Components. New York: Ronald, 1959, pp. 289-298, 371-380.

[8] Electrical Transmission and Distribution Reference Book, 4th ed. East Pittsburgh, Pa.: Westinghouse, 1964, pp. 41, 749-752.

[9] R. F. Lawrence and D. J. Povejsil, "Determination of inductive and capacitive unbalance for untransposed transmission lines," AIEE Trans. (Power Apparatus and Systems), vol. 71, pp. 547-556, April 1.952.

\section{Discussion}

W. A. Morgan (Bechtel Corporation, San Francisco, Calif.): The author brings to light a special problem that could have caused transmission planners of future systems considerable worry in some cases. The principles of his solution should serve as a way of avoiding this problem. Would the author please state what the size of the $500-\mathrm{kV}$ line shunt reactors corresponds to in terms of percent of rated line voltage charging MVA?

In many EHV transmission systems the line charging MVA must be neutralized in order to control line voltages during lineenergizing or light-load operations. Often this is accomplished by connecting a certain percentage (say, 50 percent, for example) directly to the line at line voltage without on-load switching circuit breakers, and the remainder of the shunt reactance is in the form of low-voltage shunt reactors connected to the tertiary windings of terminal transformers by means of circuit breakers. In this way, the full requirement of shunt reactance is available to compensate for the line charging MVA when required for light-load conditions, but a portion may be switched off (preferably in steps) as the load on the line builds up and would otherwise require voltage support. Perhaps the author would comment on the possibility of varying the percentage of switchable and nonswitchable shunt reactors as a means of avoiding the problem he has studied.

The author had a specific problem to solve and his conclusions are slanted toward that problem. However, it would be interesting if a simple rule or guideline might be developed to aid future planners relating to the percentage of line charging MVA compensation that should be avoided. Could we be fortunate enough that this might correspond to a 100-percent line charging MVA?

It is suggested that the author treat the subject generally and develop rules or guidelines for avoiding resonant voltage conditions of long, paralleling EHV circuits in the selection of unswitched shunt reactors.

Manuscript received June 28, 1968.

R. K. Green (Dallas Power and Light Company, Dallas, Tex. 75201): The author is to be congratulated on his technique for analyzing a very complex problem. The thoroughness of his paper is testimony to a great amount of time and labor. Individuals without access to a network analyzer may now with a digital computer solve EHV network problems taking into account the distributed characteristic of electrostatic and electromagnetic line constants.

In performing similar calculations, $I$ have found that if the reactor connected to wires 1,2 , and 3 has an impedance $Z_{n}$ connected between the reactor neutral and ground, the $Z_{a}$ of (8) becomes

$Z_{a}=\left[\begin{array}{cccccc}Z_{a 11}+Z_{n} & Z_{n} & Z_{n} & 0 & \cdots & 0 \\ Z_{n} & Z_{a 22}+Z_{n} & Z_{n} & 0 & \cdots & 0 \\ Z_{n} & Z_{n} & Z_{a 33}+Z_{n} & 0 & \cdots & 0 \\ 0 & 0 & 0 & Z_{a 44} & \cdots & 0 \\ \cdot & \cdot & \cdot & \cdot & \cdots & \cdot \\ \cdot & \cdot & \cdot & \cdot & \cdots & \cdot \\ 0 & 0 & 0 & 0 & \cdots & Z_{a n n}\end{array}\right]$.

Considering the reactor neutral impedance adds another dimension to the resonance analysis.

I wonder if Mr. Chaston would like to elaborate on his statement "... there are several resonance values, a condition which would be expected because of the various values of capacitance between the respective conductors." Has he done any work since submitting his paper in regard to the determination of the causes of the individual resonant points? Is it possible that one resonant point is due to phase-to-ground couplings, while another is due to phaseto-phase couplings, and another is due to coupling between circuits?

Manuscript received July 12, 1968. 
M. H. Hesse and D. D. Wilson (General Electric Company, Schenectady, N. Y. 12305): The inclusion of the electromagnetic parameters of the transmission line representation as described in this paper is a major step toward accurate load flow representation, as well as analyses of the effects of unsymmetrical faults on either of the coupled lines. It would be necessary, however, to consider system equivalent impedances (i.e., line terminal impedances) to accurately calculate these voltage unbalances.

In the studies discussed in [2], load flow and unbalanced faults were examined on a three-phase representation utilizing an ac network analyzer, and faults were found to be of primary importance in the production and severity of overvoltages. For example, if one of the two coupled circuits was open at both ends and a line-toground fault were placed on the energized circuit, the voltages on the de-energized circuit generally increased. Since the phenomenon is primarily of zero sequence nature, this is to be expected and must be considered in specific cases where near resonance is encountered.

Another area of interest is the effect of the differences between zero sequence and positive sequence reactance of three-phase reactor units. Although the zero sequence characteristics are (to various degrees) nonlinear, it would be of interest to ascertain trends in coupled overvoltages as a function of approximated linear zero sequence reactances. This would, of course, necessitate symmetrical component calculation procedures rather than phase equations; however, the matrices as derived by the author can be converted to symmetrical component notation with relative ease.

Invariably, problems associated with transmission line dissymmetry are not easily categorized, and therefore generalized solutions are difficult to establish with any great degree of rigor. A digital computer program, such as described by Prof. Chaston, offers the flexibility of solving the problem accurately for a wide variety of combinations of system components. Although generalized solutions for this phenomenon are difficult due to the vast number of variables, trends premised on the effects of various system faults, line configurations, equivalent source impedances, reactor characteristics, etc., would be a worthwhile endeavor, possibly for a future paper.

Prof. Chaston's results confirm the statements in [2] that transpositions materially reduce the voltage stress across shunt reactors on overhead lines. Transposition scheme 2 requires crossovers between circuits and therefore may be somewhat impractical. It should be recognized that the results of an analysis are no more accurate than the input or known system data. While the magnetic parameters of a transmission line can be defined with relatively high accuracy, the electrostatic parameters are a significant function of variations in terrain, tower structures, adjacent vegetation, etc. Theoretical results must therefore be appropriately seasoned with engineering judgment. In the final analysis, several economic alternatives exist for reducing the near resonance phenomenon, and each must be evaluated on its own merits.

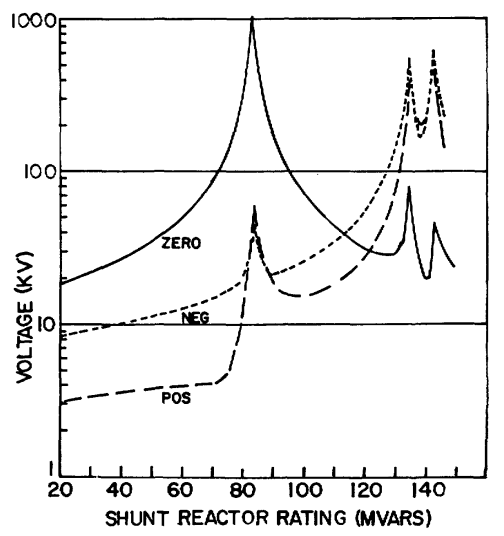

Fig. 13. Symmetrical component voltages for the Texas Electric Service Company line.

A. N. Chaston: The discussions are appreciated. Mr. Morgan inquired regarding percent of shunt reactor compensation. The value of shunt capacitance is 0.1424 megohm-miles per conductor, 712 ohms for 200 miles, or 387 MVA for 200 miles. Therefore, a shunt reactor compensation of $125 \mathrm{MVA}$ at each end of the 200-mile lines is 65 percent compensation.

$\mathrm{Mr}$. Green requested comment regarding the several resonance values. The original remark was made to emphasize the existence of multiple resonance peaks. Further study has not been completed to verify the exact cause.

Dr. Hesse and Mr. Wilson mentioned the desirability of having the program calculate in terms of symmetrical components. Shah [10] has recently obtained the data for Fig. 13. This data may be compared with the Texas Electric Service Company circuit described in [1] and [2] with specific reference to Figs. 1(b) and 3 (b) of [2]. It is interesting to note the first peak is predominantly zero sequence whereas the second peak is negative sequence.

The discussions indicate that considerable study of the topic remains to be completed. This will be done as time, funds, and personnel become available.

\section{REFERENCES}

10] N. K. Shah, "Computer analysis of a double-circuit transmission line using symmetrical components," M.S. thesis Brigham Young University, Provo, Utah, August 1948.

Manuscript received August 15, 1968. 